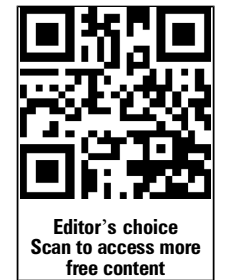

- Additional material is published online only. To view please visit the journal online (http://dx.doi.org/10.1136/ heartjnl-2013-304421).

For numbered affiliations see end of article.

\section{Correspondence to}

Dr Akira Sekikawa, Graduate School of Public Health, University of Pittsburgh, 130 North Bellefield Avenue, Suite 546, Pittsburgh, PA 15213, USA; akira@pitt.edu

Received 6 June 2013 Revised 7 November 2013 Accepted 8 November 2013 Published Online First 18 December 2013

\section{SLinked}

- http://dx.doi.org/10.1136/ heart-2013-305257

To cite: Sekikawa $A$ Miura $\mathrm{K}$, Lee $\mathrm{S}$, et al. Heart 2014;100:569-573.

\title{
Long chain n-3 polyunsaturated fatty acids and incidence rate of coronary artery calcification in Japanese men in Japan and white men in the USA: population based prospective cohort study
}

\author{
Akira Sekikawa, ${ }_{1}^{1}$ Katsuyuki Miura, ${ }^{2}$ Sunghee Lee, ${ }^{1}$ Akira Fujiyoshi, ${ }^{2}$ \\ Daniel Edmundowicz, ${ }^{3,4}$ Takashi Kadowaki, ${ }^{2}$ Rhobert W Evans, ${ }^{1}$ Sayaka Kadowaki, ${ }^{2}$ \\ Kim Sutton-Tyrrell, ${ }^{1}$ Tomonori Okamura, ${ }^{2,5}$ Marnie Bertolet, ${ }^{1}$ Kamal H Masaki, ${ }^{6}$ \\ Yasuyuki Nakamura, ${ }^{7}$ Emma J M Barinas-Mitchell, ${ }^{1}$ Bradley J Willcox, ${ }^{6}$ Aya Kadota, ${ }^{2,8}$ \\ Todd B Seto, ${ }^{9}$ Hiroshi Maegawa, ${ }^{10}$ Lewis $\mathrm{H}$ Kuller, ${ }^{1}$ Hirotsugu Ueshima, ${ }^{2}$ for the ERA \\ JUMP Study Group
}

\begin{abstract}
Objective To determine whether serum concentrations of long chain $n-3$ polyunsaturated fatty acids (LCn3PUFAs) contribute to the difference in the incidence rate of coronary artery calcification (CAC) between Japanese men in Japan and white men in the USA.
\end{abstract}

Methods In a population based, prospective cohort study, 214 Japanese men and 152 white men aged 40-49 years at baseline (2002-2006) with coronary calcium score $(C C S)=0$ were re-examined for $C A C$ in 2007-2010. Among these, 175 Japanese men and 113 white men participated in the follow-up exam. Incident cases were defined as participants with $C C S \geq 10$ at follow-up. A relative risk regression analysis was used to model the incidence rate ratio between the Japanese and white men. The incidence rate ratio was first adjusted for potential confounders at baseline and then further adjusted for serum LCn3PUFAs at baseline. Results Mean (SD) serum percentage of LCn3PUFA was $>100 \%$ higher in Japanese men than in white men (9.08 (2.49) vs 3.84 (1.79), respectively, $p<0.01$ ) Japanese men had a significantly lower incidence rate of CAC compared to white men (0.9 vs $2.9 / 100$ personyears, respectively, $p<0.01$ ). The incidence rate ratio of CAC taking follow-up time into account between Japanese and white men was $0.321(95 \% \mathrm{Cl} 0.150$ to 0.690; $p<0.01)$. After adjusting for age, systolic blood pressure, low density lipoprotein cholesterol, diabetes, and other potential confounders, the ratio remained significant $(0.262,95 \% \mathrm{Cl} 0.094$ to $0.731 ; p=0.01)$. After further adjusting for LCn3PUFAs, however, the ratio was attenuated and became non-significant $(0.376$, $95 \% \mathrm{Cl} 0.090$ to $1.572 ; p=0.18$ ).

Conclusions LCn3PUFAs significantly contributed to the difference in the incidence of CAC between Japanese and white men.

\section{INTRODUCTION}

Coronary heart disease (CHD) is a major public health problem not only in developed but also in developing countries. ${ }^{1}$ CHD rates in Japan are uniquely low compared to the USA and other developed countries. ${ }^{2}$ Even among men born in Japan after the second world war who have been exposed to a westernised lifestyle since childhood, CHD mortality in Japan is much lower than in the USA, despite a less favourable or similar profile of many cardiovascular risk factors in the Japanese, including blood pressure (BP), low density lipoprotein cholesterol (LDL-C), smoking, and type 2 diabetes mellitus (T2DM). ${ }^{3}$ The low CHD mortality in Japan is not due to misclassification of the cause of death ${ }^{4}$ or a cohort effect. ${ }^{3}$

Coronary artery calcification (CAC) is highly correlated with atherosclerotic burden of the coronary arteries. ${ }^{5}$ Studies in the general population-for example, the Coronary Artery Risk Development in Young Adult (CARDIA), ${ }^{6}$ the Multi-Ethnic Study of Atherosclerosis (MESA), ${ }^{7}$ and our study ${ }^{8}-$ reported that $\mathrm{CAC}$ is associated with traditional risk factors. Moreover, CAC is a strong independent predictor of CHD. ${ }^{9}$ We have previously reported that the prevalence of CAC in Japanese subjects was lower than that in US white subjects, whereas the prevalence in Japanese Americans was higher as compared to US white subjects, ${ }^{10}$ indicating that low CHD mortality in Japanese individuals in Japan is unlikely to be primarily due to genetic factors.

Long-chain n-3 polyunsaturated fatty acids (LCn3PUFAs) are major regulators of multiple molecular pathways among other fatty acids, causing various biological effects, including antiarrhythmic and antiatherogenic effects. ${ }^{11}$ The antiarrhythmic effect is well documented and the results of some, but not all, randomised clinical trials (RCTs) support this effect. ${ }^{12}$ Although several RCTs in patients with CHD or T2DM reported the effect of LCn3PUFAs on the progression of atherosclerosis, ${ }^{13} 14$ no previous studies have reported the association of LCn3PUFAs with the progression of atherosclerosis in the general population.

The Japanese population has a notably higher dietary intake of LCn3PUFAs compared to other populations, for example, $1000 \mathrm{mg} /$ day in Japan versus $<100 \mathrm{mg} /$ day in typical western diets, 
including the USA. ${ }^{15}$ We have previously reported in our crosssectional study that high serum concentrations of LCn3PUFAs in Japanese subjects significantly contributed to the difference in the prevalence of CAC between Japanese and US white subjects. ${ }^{10}$ In the current study, we hypothesised that the incidence rate of CAC was significantly lower in Japanese men than US white men and that higher values of LCn3PUFAs contributed to the difference in the CAC incidence rate. We tested these hypotheses in the electron beam tomography, risk factor assessment among Japanese and US men in the post-World-War-II birth cohort (ERA JUMP Study), a population based study of 868 men aged 40-49 years comprising Japanese subjects in Japan, US white subjects, and Japanese Americans.

\section{METHODS}

Study sample

The ERA JUMP Study is an international, population based study of atherosclerosis in men aged 40-49 years at baseline. A detailed description of the study design and methods has been published previously. ${ }^{10}$ Briefly, 313 Japanese men from Kusatsu, Shiga, Japan, 310 white men from Allegheny County, Pennsylvania, USA, and 303 Japanese American men from Honolulu, Hawaii, USA from a representative sample of offspring of fathers who participated in the Honolulu Heart Program, were examined at baseline between 2002 and 2006. All participants were without clinical cardiovascular disease. The recruitment of the follow-up study in the Japanese and white subjects was started in 2007 and was completed in 2010, whereas the follow-up in the Japanese Americans subjects was started in 2009 and is ongoing. Thus, the data presented in the current study excludes the Japanese Americans.

All participants from the baseline examination were invited to the follow-up. The rates of participation were $83.7 \%$ in the Japanese men (262/313) and 79.0\% in the white men (245/ 310). Individuals with missing CAC $(n=9)$ were excluded, resulting in a total of 498 participants (255 Japanese and 243 white men). The characteristics of the participants and nonparticipants were not significantly different (see online supplementary table S1).

The current study analysed the incidence rather than the progression of CAC because the prevalence of CAC, defined as a CCS $\geq 10$, was low (9.3\%) in the Japanese subjects at baseline. ${ }^{10}$ Incident cases were defined as individuals with a CCS $=0$ at baseline (214 Japanese and 152 white men) and a CCS $\geq 10$ at follow-up. We used CCS $\geq 10$ because $54 \%$ of the Japanese subjects $(27 / 50)$ and $28 \%$ of the white subjects $(19 / 67)$ with CCS $>0$ and $<10$ at baseline reverted to $\mathrm{CCS}=0$ at follow-up, whereas only three Japanese subjects and two white subjects with CCS $\geq 10$ reverted to CCS $=0$ at follow-up (see online supplementary table S2). Thus, the final sample comprised 175 Japanese and 113 white men with $C C S=0$ at baseline. Informed consent was obtained from all participants. The study was approved by the institutional review boards of the Shiga University of Medical Science, Otsu, Japan and the University of Pittsburgh, Pittsburgh, USA.

\section{Coronary artery calcification}

CAC was measured using electron beam tomography (EBT) (Imatron C150, GE Medical Systems, South San Francisco, USA) at baseline both in Japan and in the USA, and follow-up in the USA. ${ }^{10}$ At follow-up in Japan, CAC was measured using a 16 slice multidetector CT (MDCT) (Toshiba Medical System Corporation, Tochigi, Japan). Scanners were calibrated regularly by technicians following a standardised protocol. A total of 30-
40 contiguous $3 \mathrm{~mm}$ thick transverse images were obtained from the level of the aortic root to the apex of the heart. Images were recorded during a maximal breath hold using ECG guided triggering. CAC was considered to be present with three contiguous pixels $\left(\right.$ area $\left.=1 \mathrm{~mm}^{2}\right) \geq 130$ Hounsfield units. One trained reader at the Cardiovascular Institute, University of Pittsburgh, read the images using a Digital-Imaging-andCommunications-in-Medicine workstation and software by the AccuImage Diagnostic Corporation, San Francisco, which calculates CCS with the Agatston scoring method. ${ }^{10}$ The reader was blinded to each participant's characteristics and the study centres. The intra-reader reproducibility of non-zero CCSs had an intra-class correlation of 0.98 .

\section{Serum LCn3PUFAs and other covariates}

All participants underwent a physical examination, lifestyle questionnaire, and laboratory assessment as described previously. ${ }^{10} \mathrm{BP}$ was measured in the right arm of the seated participant after the participant emptied his bladder and sat quietly for $5 \mathrm{~min}$, using an appropriate sized cuff, with an automated sphygmomanometer (BP-8800, Colin Medical Technology, Komaki, Japan). The average of two measurements was used. T2DM was defined as an individual with fasting glucose $\geq 7.0 \mathrm{mmol} / \mathrm{L}$ or under diabetic medication. Alcohol drinkers were defined as those who drank alcohol $\geq 2$ days/week.

Venepuncture was performed early in the clinic visit after a 12 $\mathrm{h}$ fast. Serum and plasma samples were stored at $-80^{\circ} \mathrm{C}$, shipped on dry ice to the University of Pittsburgh to determine concentrations of LDL-C, high density lipoprotein cholesterol (HDL-C), triglycerides, glucose, and $\mathrm{C}$ reactive protein (CRP). Gas chromatography was used to determine serum n-3 and other fatty acid content which is expressed as a percentage of serum total fatty acids. The coefficients of variation between runs for major marine-derived n-3 fatty acids, eicosapentaenoic acid (20:5n-3) (EPA) and docosahexaenoic acid (22:6n-3) (DHA), were 4.5\% and $7.2 \%$, respectively, as previously reported. ${ }^{10}$

\section{Statistical methods}

An incidence rate was calculated as the number of incident cases divided by the number of the population exposed to risk between baseline and follow-up scans (per 100 person-years). To compare baseline characteristics between Japanese and white subjects as well as between incident and non-incident cases in each of the Japanese and white men, $t$ test or Mann-Whitney $\mathrm{U}$ test for continuous variables and $\chi^{2}$ test for categorical variables were used.

A relative risk regression analysis was used to model the incidence rate ratio between the Japanese and white subjects after adjusting for potential confounders, using a generalised linear model with log link and binomial error distribution. First, we showed a relative risk in an unadjusted model which took follow-up time into account. Then, in model I, we adjusted for age, systolic BP, LCL-C, HDL-C, triglycerides, diabetes, body mass index (BMI), pack-years of smoking, and medication for hypertension based on previous literature. ${ }^{16} 17$ Finally, in model II we further adjusted for total LCn3PUFAs, which were defined as the sum of EPA, DHA, and docosapentaenoic acid. Values of $\mathrm{p}<0.05$ were considered to indicate statistical significance. All these statistical analyses were performed with Stata V.11.2 (StataCorp LP, College Station, Texas, USA).

\section{RESULTS}

Baseline characteristics show that age, systolic BP, LDL-C, triglycerides, and rates of T2DM were not significantly different 
between the two groups $(p>0.10)$. Compared to the white men, the Japanese men had significantly higher rates of both current and former smokers and higher values of glucose $(\mathrm{p}<0.05)$, although the Japanese men had significantly lower BMI and CRP values, and significantly higher concentrations of HDL-C $(\mathrm{p}<0.05)$. Mean percentages of total LCn3PUFAs, EPA, and DHA were more than $100 \%$ higher in the Japanese than in the white subjects $(p<0.01)$. Among these individuals, 10 Japanese and 15 white men had a CCS $\geq 10$ at follow-up, and the incidence rate of CAC was significantly lower in the Japanese than in the white subjects $(p<0.01)$ (table 1$)$.

Table 2 shows the risk factors and other covariates between the incident and non-incident cases in each group. These factors did not differ significantly between Japanese and white subjects $(p>0.10)$. The only exception was systolic BP in the Japanese men, which was significantly higher in incident cases than in non-incident cases $(\mathrm{p}<0.01)$.

The incidence rate ratio of CAC between the Japanese and white men was $0.321(\mathrm{p}<0.01)$ in an unadjusted model (table 3$)$. After adjusting for systolic BP, LCL-C, HDL-C, triglycerides, diabetes, BMI, pack-years of smoking, and medication for hypertension, the incidence rate ratio remained significant (model I in table 3). After further adjusting for total LCn3PUFAs, the

Table 1 Characteristics of participants with zero coronary calcium score at baseline, number of incidence cases and incidence rates in Japanese and white subjects

\begin{tabular}{|c|c|c|}
\hline & $\begin{array}{l}\text { Japanese subjects } \\
(\mathrm{n}=175)\end{array}$ & $\begin{array}{l}\text { White subjects } \\
(n=113)\end{array}$ \\
\hline Age (years) & $44.9(2.8)$ & $44.7(2.9)$ \\
\hline Body mass index $\left(\mathrm{kg} / \mathrm{m}^{2}\right)$ & $23.2(2.8)$ & $26.3(3.0)$ \\
\hline Systolic blood pressure $(\mathrm{mm} \mathrm{Hg})$ & $124.0(15.3)$ & $121.6(11.0)$ \\
\hline Medication for hypertension (\%) & 4.0 & 5.3 \\
\hline LDL-C (mmol/L) & $3.33(0.95)$ & $3.45(0.81)$ \\
\hline HDL-C (mmol/L) & $1.41(0.36)$ & $1.25(0.30)$ \\
\hline Triglycerides (mmol/L) & $1.48(1.08,1.97)$ & $1.36(1.02,1.87)$ \\
\hline Medication for hyperlipidaemia (\%) & 1.7 & 8.0 \\
\hline Glucose (mmol/L) & $5.81(0.69)$ & $5.52(0.47)$ \\
\hline Diabetes (\%) & 4.6 & 1.8 \\
\hline $\mathrm{C}$ reactive protein (mg/L) & $0.30(0.15,0.65)$ & $0.83(0.44,1.65)$ \\
\hline \multicolumn{3}{|l|}{ Smoking } \\
\hline Current $(\%)$ & 48.6 & 8.0 \\
\hline Former (\%) & 32.0 & 18.6 \\
\hline Pack years & $18.0(3.0,29.0)$ & $0.0(0.0,1.6)$ \\
\hline \multicolumn{3}{|l|}{ Alcohol } \\
\hline Drinker ( $\geq 2$ days/week) & 68.0 & 54.9 \\
\hline Ethanol consumption ( $\mathrm{g} /$ day) & $14.0(2.9,38.0)$ & $8.2(1.6,18.7)$ \\
\hline Total LCn3PUFAs (\%) & $9.08(2.49)$ & $3.84(1.79)$ \\
\hline EPA (\%) & $2.36(1.12)$ & $0.79(0.63)$ \\
\hline DHA (\%) & $5.88(1.51)$ & $2.38(1.24)$ \\
\hline Number of incident cases & 10 & 15 \\
\hline Follow-up time (years) & $6.2(0.4)$ & $4.6(0.2)$ \\
\hline Incidence rate (/100 person years) & 0.9 & 2.9 \\
\hline
\end{tabular}

Values are expressed as mean (SD) or median (25th and 75th centile) for continuous variables and $\%$ for categorical variables.

Total LCn3PUFAs were defined as the sum of eicosapentaenoic, docosapentaenoic, and docosahexaenoic acids. Fatty acids were expressed as a percentage of total serum fatty acids.

DHA, docosahexaenoic acid; EPA, eicosapentaenoic acid; HDL-C, high density lipoprotein cholesterol; LCn3PUFA, long chain n-3 polyunsaturated fatty acid; LDL-C, low density lipoprotein cholesterol. statistical significant difference in incidence rate ratio was attenuated and became non-significant (model II in table 3 ).

\section{DISCUSSION}

This international study has shown that the CAC incidence rate was significantly lower in the Japanese men than in US white men, and that the significant difference in the CAC incidence rate, which remained after adjusting for risk factors and potential confounders, became non-significant after further adjusting for serum LCn3PUFAs. The results of the longitudinal study extended our previous findings from our cross-sectional study, ${ }^{10}$ suggesting that high dietary intake of LCn3PUFAs contributed to the low levels of atherosclerosis in Japanese subjects.

Results from two recent large studies in Japan support the antiatherogenic effect of LCn3PUFAs. ${ }^{18} 19$ The Japan EPA Lipid Intervention Study (JELIS) tested a hypothesis that long term supplementation with $1800 \mathrm{mg} /$ day EPA prevented major coronary events in 18645 hypercholesterolaemic individuals. ${ }^{18}$ After a mean follow-up of 4.6 years, a 19\% significant relative reduction in the primary end point occurred in the EPA group. Similarly, a 10 year longitudinal population based study in Japan of 41578 individuals aged 40-59 years reported that dietary intake of LCn3PUFAs had a significant inverse association with non-fatal coronary events. ${ }^{19}$

In contrast, several recent RCTs of LCn3PUFAs on cardiovascular disease-that is, SU.FOL.OM $3,{ }^{20} \alpha$ Omega Trail, ${ }^{21}$ ORIGIN, ${ }^{22}$ and the Risk and Prevention Study ${ }^{23}$-failed to show their beneficial effect. However, doses of LCn3PUFAs used in these RCTs were much lower than the dose in JELIS: 376-900 mg/day vs $1800 \mathrm{mg} /$ day, respectively. Moreover, background dietary intake of LCn3PUFAs in these populations is substantially lower than that in Japan. Therefore, these results from recent RCTs do not address the question of whether LCn3PUFAs at concentrations observed in Japanese subjects have an antiatherogenic effect.

We defined incident cases of CAC as those with CCS $=0$ at baseline and CCS $\geq 10$ at follow-up. This is because we cannot deny the possibility that CCSs of 1-9 are a noise. Indeed, a substantial proportion of individuals with CCSs $>0$ and $<10$ at baseline reverted to $\mathrm{CCS}=0$ at follow-up $(39 \% \quad(46 / 117))$. Inter-scan variability of CCS is much higher when CCSs are $>0$ and $<10$ as compared to CCSs $\geq 10$. $^{24}$ For this reason, the Dallas Heart Study used CCS $\geq 10$ as indicating the presence of CAC. $^{24}$ Although CARDIA and MESA defined incident cases of CAC as individuals with $\mathrm{CCA}=0$ at baseline and CCS $>0$ at follow-up, a noise caused by inter-scan variability in these studies is expected to be lower than our study; this is because these studies scanned twice at both baseline and follow-up, ${ }^{25}$ whereas our study scanned once both at baseline and follow-up.

The effect of LCn3PUFAs on cardiovascular risk factors has been documented in RCTs and experimental studies showing that LCn3PUFAs significantly lower triglycerides and BP, nonsignificantly raise HDL-C, and have inconsistent effects on LDL-C, glucose, and CRP. ${ }^{11} 2627$ The antiatherogenic effect of LCn3PUFAs is, however, unlikely to be mediated through these factors in the current study; this is because the difference in the incidence rate ratio of CAC remained significant after adjusting for these factors but became non-significant after further adjusting for LCn3PUFAs. Experimental studies show that LCn3PUFAs have various antiatherogenic properties including suppressing the production of inflammatory cytokines, the expression of cell adhesion molecules, and improving endothelial function, platelet function, and plaque stability. ${ }^{11} 27$ Additionally, long term exposure to high doses of LCn3PUFAs 
Table 2 Description of risk factors between incident and non-incident subjects in Japanese and white men

\begin{tabular}{|c|c|c|c|c|}
\hline & \multicolumn{2}{|c|}{ Japanese subjects ( $n=175$ ) } & \multicolumn{2}{|c|}{ White subjects $(n=113)$} \\
\hline & Incident $(n=10)$ & Non-incident $(n=165)$ & Incident $(n=15)$ & Non-incident $(n=98)$ \\
\hline Age (years) & $45.4(3.6)$ & $44.9(2.8)$ & $44.2(2.9)$ & $44.7(2.9)$ \\
\hline Body mass index $\left(\mathrm{kg} / \mathrm{m}^{2}\right)$ & $22.8(1.7)$ & $23.2(2.9)$ & $27.7(3.1)$ & $26.2(3.0)$ \\
\hline Systolic blood pressure (mm Hg) & $140.6(24.0)$ & $123.0(14.0)$ & $122.7(9.6)$ & $121.4(11.2)$ \\
\hline Hypertension medication (\%) & 10.0 & 3.6 & 6.7 & 5.1 \\
\hline LDL-C (mmol/L) & $3.13(1.13)$ & $3.34(0.95)$ & $3.26(0.77)$ & $3.48(0.82)$ \\
\hline $\mathrm{HDL}-\mathrm{C}(\mathrm{mmol} / \mathrm{L})$ & $1.42(0.35)$ & $1.41(0.36)$ & $1.13(0.20)$ & $1.27(0.31)$ \\
\hline Triglycerides (mg/dL) & $1.46(1.31,1.64)$ & $1.48(1.08,1.98)$ & $1.27(1.08,1.91)$ & $1.36(0.99,1.87)$ \\
\hline Medication for hyperlipidaemia (\%) & 0 & 1.8 & 0 & 9.2 \\
\hline Glucose (mmol/L) & $5.83(0.64)$ & $5.81(0.70)$ & $5.47(0.40)$ & $5.53(0.48)$ \\
\hline Diabetes (\%) & 10.0 & 4.2 & 2.0 & 0 \\
\hline $\mathrm{C}$ reactive protein (mg/L) & $0.3(0.2,07)$ & $0.3(0.2,0.7)$ & $1.0(0.7,1.8)$ & $0.8(0.4,1.6)$ \\
\hline \multicolumn{5}{|l|}{ Smoking } \\
\hline Current smokers (\%) & 60.0 & 47.9 & 0 & 9.2 \\
\hline Former smokers (\%) & 30.0 & 32.1 & 20.0 & 18.4 \\
\hline Pack years of smoking & $21.3(4.6,44.1)$ & $18.0(2.9,28.3)$ & $0(0,0)$ & $0(0,2.1)$ \\
\hline \multicolumn{5}{|l|}{ Alcohol } \\
\hline Alcohol drinker (\%) & 80.0 & 67.3 & 53.3 & 55.1 \\
\hline Ethanol consumption ( $\mathrm{g} /$ day) & $36.0(17.0,51.4)$ & $14.0(2.3,37.0)$ & $6.2(0.8,27.8)$ & $8.7(1.6,18.6)$ \\
\hline Marine $n-3$ fatty acids (\%) & $8.5(2.6)$ & $9.1(2.5)$ & $3.47(1.76)$ & $3.90(1.80)$ \\
\hline EPA (\%) & $1.9(0.8)$ & $2.4(1.1)$ & $0.81(0.65)$ & $0.72(0.53)$ \\
\hline DHA (\%) & $5.7(1.8)$ & $5.9(1.5)$ & $2.11(1.27)$ & $2.42(1.24)$ \\
\hline Follow-up (years) & $6.3(0.3)$ & $6.2(0.4)$ & $4.7(0.1)$ & $4.6(0.2)$ \\
\hline
\end{tabular}

observed in the Japanese population is likely to have effects on immune cell and gene expressions, such as inhibiting the activation of nuclear factor $\kappa-\mathrm{B}$, which in turn results in reduced expression of genes encoding proteins involved in inflammation. $^{11} 27$ It is speculated that many of these antiatherogenic actions of LCn3PUFAs most likely require their intake to be $\geq 1$ g/day. ${ }^{11}$

\section{STUDY LIMITATIONS}

The current study has several limitations. The number of CAC incident cases was small and our univariate analyses were unable to identify factors significantly associated with incident cases except for systolic BP in the Japanese subjects. The current study examined men aged 40-49 years at baseline and the results may not be generalisable to older age groups or women. However, we chose this age group specifically because, unlike

Table 3 Incidence rate ratio of coronary artery calcification between Japanese and white subjects

\begin{tabular}{llr}
\hline & Incidence rate ratio $(95 \% \mathrm{Cl})$ & $\mathrm{p}$ Value \\
\hline Unadjusted model & $0.321(0.150$ to 0.690$)$ & $<0.01$ \\
Model I & $0.262(0.094$ to 0.731$)$ & 0.01 \\
Model II & $0.376(0.090$ to 1.572$)$ & 0.18 \\
\hline
\end{tabular}

Unadjusted model took the follow-up time into account.

Model I: adjusted for age, systolic blood pressure, LDL-C, HDL-C, triglycerides, body

mass index, diabetes, pack-years of smoking, and medication for hypertension.

Model II: further adjusted for total LCn3PUFAs in addition to model I.

HDL-C, high density lipoprotein cholesterol; LCn3PUFA, long-chain n-3

polyunsaturated fatty acid; LDL-C, low density lipoprotein cholesterol. older age groups, levels of serum total cholesterol and BP have been similar between the Japanese men and US white men throughout their lifetime in this birth cohort. ${ }^{8}$ Although EBT was used at baseline both in the USA and Japan, and at follow-up in the USA, MDCT was used at follow-up in Japan. Moreover, the current study did not use an external standard to calibrate CCS between EBT and MDCT in Japan. Since MDCT as compared to EBT may be less sensitive in detecting low $\mathrm{CCSs}^{28}{ }^{28}$ our analysis defining incident cases as those with CCSs $\geq 10$ at follow-up should be more robust than analysis defining incidence cases as those with CCSs $>0$ at follow-up. Although individuals with CCSs $\geq 10$ are reported to have a significant association with all cause mortality as compared to individuals with $\mathrm{CCS}=0,{ }^{29}$ incident cases of CAC are not a clinical outcome. Because the study is observational, our finding might be due to unmeasured confounders. Serum LCn3PUFAs reflect short term dietary fat intake and may not reflect long term dietary intake. ${ }^{30}$ However, because the variation in serum LCn3PUFAs occurs randomly, the actual association of LCn3PUFAs with CAC incidence is likely to be stronger than was observed in the current study.

\section{CONCLUSIONS}

In summary, the present study has shown that the CAC incidence rate was significantly lower in Japanese men than in US white men and that this difference cannot be explained by differences in known risk factors. It did, however, became nonsignificant after further adjusting for serum LCn3PUFAs. These results may suggest the antiatherogenic effect of LCn3PUFAs at values observed in Japanese subjects. 
Key messages

What is already known about this subject? Much higher serum values of long chain $n-3$ polyunsaturated fatty acids (LCn3PUFAs) in Japanese men in Japan than in the USA significantly contribute to the cross-sectional difference in atherosclerosis evaluated by coronary artery calcification (CAC) and intima-media thickness of the carotid artery; both are independent predictors of future cardiovascular events in men in Japan and the USA.

\section{What does this study add?}

Serum values of $L C n 3 P$ PUFAs in Japanese subjects in Japan significantly contributed to the longitudinal difference in CAC (ie, incidence of CAC) between men in Japan and the USA, independent of other cardiovascular risk factors.

How might this impact on clinical practice? Higher doses of LCn3PUFAs than that used in recent randomised clinical trials of LCn3PUFAs on cardiovascular disease may potentially have beneficial effects on cardiovascular disease.

\section{Author affiliations}

'Department of Epidemiology, University of Pittsburgh, Pittsburgh, Pennsylvania, USA

${ }^{2}$ Department of Health Science, Shiga University of Medical Science, Otsu, Shiga, Japan

${ }^{3}$ Department of Medicine, Temple University, Philadelphia, Pennsylvania, USA

${ }^{4}$ Heart and Vascular Institute, University of Pittsburgh Medical Center, Pittsburgh, Pennsylvania, USA

${ }^{5}$ Department of Preventive Medicine and Public Health, Keio University, Tokyo, Japan ${ }^{6}$ Department of Geriatric Medicine, University of Hawaii, Honolulu, Hawaii, USA ${ }^{7}$ Department of Cardiovascular Epidemiology, Kyoto Women's University, Kyoto, Japan

${ }^{8}$ Osaka Kyoiku University, Kashiwara, Osaka, Japan

${ }^{9}$ Internal Medicine \& Cardiology, Queen's Hospital, Honolulu, Hawaii, USA

${ }^{10}$ Department of Medicine, Shiga University of Medical Science, Otsu, Shiga, Japan

Contributors SA, MK, ED, KT, S-TK, OT, MKH, B-ME, WJ, STB, MH, KLH, and UH mainly designed the research including project conception, development of overall research plan, and study oversight. SA, MK, FA, ED, KT, KS, ERW, KS, S-TK, OT, NY, $\mathrm{KA}, \mathrm{STB}, \mathrm{MH}$, and $\mathrm{UH}$ mainly conducted research. SA, LS, and BM analysed data or performed statistical analysis. SA and LS wrote the paper. All the above authors participated in critically revising the manuscript. SA had primary responsibility for final content. All authors read and approved the final manuscript.

Funding The work was supported by grants HL068200, HL071561, UL1RR024153, and UL1TR00005 from the National Institutes of Health, USA (Bethesda, Maryland, USA), B 16790335 and A 13307016, 17209023, and 21249043 from the Japanese Ministry of Education, Culture, Sports, Science and Technology (Tokyo, Japan).

\section{Competing interests None.}

Ethics approval Institutional review boards of Shiga University of Medical Science, Otsu, Japan and University of Pittsburgh, Pittsburgh, USA.

Patient consent Obtained.

Provenance and peer review Not commissioned; externally peer reviewed.

\section{REFERENCES}

1 Deaton C, Froelicher ES, Wu LH, et al. The global burden of cardiovascular disease. Eur I Cardiovasc Nurs 2011;10:S5-13.

2 Ueshima H, Sekikawa A, Miura K, et al. Cardiovascular disease and risk factors in Asia: a selected review. Circulation 2008;118:2702-9.

3 Sekikawa A, Horiuchi BY, Edmundowicz D, et al. A "natural experiment" in cardiovascular epidemiology in the early 21st century. Heart 2003;89:255-7.

4 Sekikawa A, Satoh T, Hayakawa T, et al. Coronary heart disease mortality among men aged 35-44 years by prefecture in Japan in 1995-1999 compared with that among white men aged 35-44 by state in the United States in 1995-1998: vital statistics data in recent birth cohort. Jpn Circ J 2001;65:887-92.

5 Greenland P, Bonow RO, Brundage BH, et al. ACCF/AHA 2007 clinical expert consensus document on coronary artery calcium scoring by computed tomography in global cardiovascular risk assessment and in evaluation of patients with chest pain. A report of the American College of Cardiology Foundation Clinical Expert Consensus Task Force (ACCF/AHA Writing Committee to Update the 2000 Expert Consensus Document on Electron Beam Computed Tomography). Circulation 2007;115:402-26.

6 Bild DE, Folsom AR, Lowe LP, et al. Prevalence and correlates of coronary calcification in black and white young adults : the Coronary Artery Risk Development in Young Adults (CARDIA) study. Arterioscler Thromb Vasc Biol 2001;21:852-7.

7 McClelland RL, Chung H, Detrano R, et al. Distribution of coronary artery calcium by race, gender, and age: results from the Multi-Ethnic Study of Atherosclerosis (MESA). Circulation 2006;113:30-7.

8 Sekikawa A, Ueshima H, Kadowaki T, et al. Less subclinical atherosclerosis in Japanese men in Japan than in white men in the United States in the post-World War II birth cohort. Am J Epidemiol 2007;165:617-24.

9 Detrano R, Guerci AD, Carr JJ, et al. Coronary calcium as a predictor of coronary events in four racial or ethnic groups. N Engl I Med 2008;358:1336-45.

10 Sekikawa A, Curb JD, Ueshima $H$, et al. Marine-derived n-3 fatty acids and atherosclerosis in Japanese, Japanese-American, and white men: a cross-sectional study. J Am Coll Cardiol 2008;52:417-24.

11 Calder PC, Yaqoob P. Marine omega-3 fatty acids and coronary heart disease. Curr Opin Cardiol 2012;27:412-19.

12 Kotwal $S$, Jun $M$, Sullivan $D$, et al. Omega 3 fatty acids and cardiovascular outcomes: systematic review and meta-analysis. Circ Cardiovasc Qual Outcomes 2012:5:808-18.

13 Angerer $\mathrm{P}$, Kothny W, Stork $\mathrm{S}$, et al. Effect of dietary supplementation with omega-3 fatty acids on progression of atherosclerosis in carotid arteries. Cardiovasc Res 2002;54:183-90.

14 Mita T, Watada H, Ogihara T, et al. Eicosapentaenoic acid reduces the progression of carotid intima-media thickness in patients with type 2 diabetes. Atherosclerosis 2007;191:162-7.

15 Stamler J, Elliott $\mathrm{P}$, Chan Q, et al. Intermap appendix tables. J Hum Hypertension 2003;17:665-775.

16 Kronmal RA, McClelland RL, Detrano R, et al. Risk factors for the progression of coronary artery calcification in asymptomatic subjects: results From the Multi-Ethnic Study of Atherosclerosis (MESA). Circulation 2007;115:2722-30.

17 Stewart JC, Zielke DJ, Hawkins MA, et al. Depressive symptom clusters and 5-year incidence of coronary artery calcification: the coronary artery risk development in young adults study. Circulation 2012;126:410-17.

18 Yokoyama M, Origasa $\mathrm{H}$, Matsuzaki $\mathrm{M}$, et al. Effects of eicosapentaenoic acid on major coronary events in hypercholesterolaemic patients (JELIS): a randomised open-label, blinded endpoint analysis. Lancet 2007;369:1090-8.

19 Iso H, Kobayashi M, Ishihara J, et al. Intake of fish and n3 fatty acids and risk of coronary heart disease among Japanese: the Japan Public Health Center-Based (JPHC) study cohort I. Circulation 2006;113:195-202.

20 Galan P, Kesse-Guyot E, Czernichow S, et al. Effects of B vitamins and omega 3 fatty acids on cardiovascular diseases: a randomised placebo controlled trial. $B M J$ 2010;341:c6273.

21 Kromhout D, Giltay EJ, Geleijnse JM. n-3 fatty acids and cardiovascular events after myocardial infarction. N Engl J Med 2010;363:2015-26.

22 The ORIGIN Trial Investigators. n-3 fatty acids and cardiovascular outcomes in patients with dysglycemia. N Engl I Med 2012.

23 Roncaglioni MC, Tombesi M, Avanzini F, et al. $\mathrm{n}-3$ fatty acids in patients with multiple cardiovascular risk factors. N Engl J Med 2013;367:309-18.

24 Jain T, Peshock R, McGuire DK, et al. African Americans and Caucasians have a similar prevalence of coronary calcium in the Dallas Heart Study. J Am Coll Cardiol 2004;44:1011-17.

25 Carr JJ, Nelson JC, Wong ND, et al. Calcified coronary artery plaque measurement with cardiac CT in population-based studies: standardized protocol of Multi-Ethnic Study of Atherosclerosis (MESA) and Coronary Artery Risk Development in Young Adults (CARDIA) study. Radiology 2005;234:35-43.

26 Balk EM, Lichtenstein AH, Chung M, et al. Effects of omega-3 fatty acids on serum markers of cardiovascular disease risk: a systematic review. Atherosclerosis 2006;189:19-30

27 De Caterina R. n-3 fatty acids in cardiovascular disease. N Engl J Med 2011;364:2439-50

28 Budoff MJ, Achenbach S, Blumenthal RS, et al. Assessment of coronary artery disease by cardiac computed tomography. Circulation 2006;114:1761-91.

29 Blaha M, Budoff MJ, Shaw LJ, et al. Absence of coronary artery calcification and all-cause mortality. JACC Cardiovasc Imaging 2009;2:692-700.

30 Hodson L, Skeaff CM, Fielding BA. Fatty acid composition of adipose tissue and blood in humans and its use as a biomarker of dietary intake. Prog Lipid Res 2008;47:348-80. 